\title{
Discrete Tomography Reconstruction Algorithms for Images with a Blocking Component
}

\author{
Stefano Bilotta and Stefano Brocchi \\ Dipartimento di Matematica e Informatica 'U. Dini', Università di Firenze, \\ Viale Morgagni, 65 - 50134 Firenze, Italy \\ \{stefano.bilotta, stefano.brocchi\}@unifi.it
}

\begin{abstract}
We study a problem involving the reconstruction of an image from its horizontal and vertical projections in the case where some parts of these projections are unavailable. The desired goal is to model applications where part of the x-rays used for the analysis of an object are blocked by particularly dense components that do not allow the rays to pass through the material. This is a common issue in many tomographic scans, and while there are several heuristics to handle quite efficiently the problem in applications, the underlying theory has not been extensively developed. In this paper, we study the properties of consistency and uniqueness of this problem, and we propose an efficient reconstruction algorithm. We also show how this task can be reduced to a network flow problem, similarly to the standard reconstruction algorithm, allowing the determination of a solution even in the case where some pixels of the output image must have some prescribed values.
\end{abstract}

\section{Introduction}

Discrete tomography is the discipline that studies the reconstruction of discrete sets from the partial information deriving from their projections. Its main motivation arises from applications that tackle the problem of obtaining information about an object by examining the data obtained from the x-rays projected through the material, as in medical scans. Differently from computerized tomography, in discrete tomography we suppose that the pixels forming the original image may have a limited set of discrete values, often only 0 or 1 . This assumption is reasonable in many cases where the object has a uniform density, and allows the definition of efficient algorithms even upon availability of a limited number of x-rays, as in 6 .

In applications, often heuristic algorithms allow an efficient reconstruction, but on the other hand determining exact algorithms for discrete tomography reconstruction is often a hard task, as the involved problems in many cases are highly undetermined or computationally intractable [12. To tackle these problems and to include some other information that may model effectively properties of the image to be rebuilt, often discrete tomography problems include

E. Barcucci et al. (Eds.): DGCI 2014, LNCS 8668, pp. 250-261 2014.

(C) Springer International Publishing Switzerland 2014 
some prior knowledge that gives birth to many variations of the reconstruction problem. Some examples that have been studied in literature include connectivity and convexity 42 , cell coloring 103 or skeletal properties 1415 . Often, with appropriate assumptions, the arising problems result to be connected to other fields of study as timetabling [18], image compression [1], network flow [5], graph theory [7] and combinatorics [13]. An excellent survey that can be examined for some classical results is [16].

In this paper, we model a situation where, due to causes such as a very dense block of material, some x-rays are blocked and do not provide measurements for these zones of the object. This is a common problem in many tomographical applications that may cause artifacts in the rebuilt images, that in some cases may reduce the quality of the reconstruction up to the point of making the resulting image unusable, for example for diagnostic purposes in the case of a medical scan. Many heuristics exists to reduce such artifacts, based on algebraic approaches [20], statistical analysis [8, linear interpolation [17, partial differential equations [9], and image impainting [11. The described approaches are often sufficient in applications, however to the best of our knowledge there is no theoretical study that determines the basic properties of consistency and uniqueness of these problems. In this paper, we answer these questions and we propose an efficient reconstruction algorithm that uses the horizontal and vertical projections. Differently from the cited works, this article does not have an immediate practical application, but is aimed to develop the theory underlying these problems. Here we consider as input of the problem projections in only two directions; this assumption is quite typical in discrete tomography, as the reconstruction problem of binary matrices from three of more projections is know to be NP-complete [12].

The paper is organized as follows. In Section 2 we give some preliminaries and describe the adopted notation. In Section 3 we describe some basic properties of the problem, and how they relate to the classical reconstruction problems without the blocking component. In Section 4 we describe an efficient reconstruction algorithm for the problem. In Section 5 we show how the reconstruction is connected with a flow problem; this result is a natural extension of the well known reduction of the standard problem, and allows us to solve the reconstruction even in the cases where some zones of the resulting image must be fixed to some prescribed values. Finally, in Section [6] we discuss and draw some conclusions with an insight on future developments.

\section{$2 \quad$ Notation and Preliminaries}

In this section we give a formal description of the studied problem. Classically, a solution of a reconstruction problem is represented by a binary matrix containing the values 0 and 1 ; we refer to the entries of the matrix as cells. In this paper, we suppose that for some zones of an image the horizontal and vertical projections that would account for some zones are unavailable, as if in a tomographical scan the area corresponding to these pixels blocked the x-rays. We refer to this set 
of cells as a blocking component; the image that we aim to rebuild hence also contains a special symbol $*$ in correspondence to the cells in this component.

Without loss of generality, the blocking component can be positioned in the last columns and rows of the matrix, as we could relate to this situation any other configuration by a rearrangement of the columns and of the rows. We also suppose that the blocking component has a rectangular shape, as any other shape would introduce some cells that do not contribute to neither the horizontal nor the vertical projections, and that are not relevant to the formulation of the problem. The problem is hence defined as follows:

Input: two integers $k^{h}$ and $k^{v}$ representing the size of the blocking component, and two vectors of projections $H=\left(h_{1}, \ldots, h_{n-k^{v}}\right), V=\left(v_{1}, \ldots, v_{m-k^{h}}\right)$.

Output: an $n \times m$ matrix $A=\left(a_{i, j}\right)$, such that:

- $\forall 1 \leq i \leq n-k^{v}, \sum_{j=1}^{m} a_{i, j}=h_{i}$;

- $\forall 1 \leq j \leq m-k^{h}, \sum_{i=1}^{n} a_{i, j}=v_{j}$;

- $\forall a_{i, j}: i>n-k^{v}, j>m-k^{h}$ then $a_{i, j}=*$.

An interesting feature of our problem is that, due to the missing projections, the sum of the horizontal and vertical projections may differ, but even in this case, there may be a solution fitting the constraints. We define this difference as $D=\sum_{j} v_{j}-\sum_{i} h_{i}$, and without loss of generality we consider $D \geq 0$. In Figure 1], we depicted an example of the problem with horizontal and vertical projections, and with a blocking component covering two rows and two columns. The vectors $H$ and $V$ represent the input of the problem, while the content of the cells of the matrix represents one of the possible solutions.

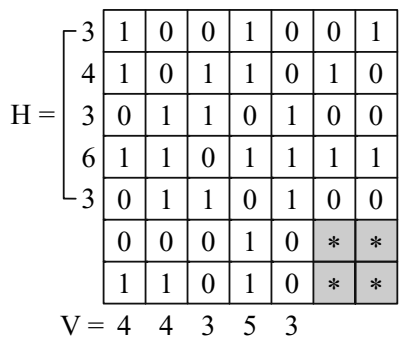

Fig. 1. An instance of the considered problem and a possible solution

\section{Properties}

From classical results in literature, we dispose of a theorem which guarantees the existence of a solution of the standard reconstruction problem. In this case, we want to rebuild a binary matrix with horizontal and vertical projections equal 
to $H$ and $V$. Without loss of generality, hereafter we consider the vectors $H$ and $V$ to be ordered, so $h_{1} \geq h_{2} \geq h_{3} \ldots$ and $v_{1} \geq v_{2} \geq v_{3} \ldots$

Definition 1. For two vectors $A, B$ of length $n$, then $A \leq_{d} B$ if for every $j$ we have $\sum_{i=1}^{j} a_{i} \leq \sum_{i=1}^{j} b_{i}$.

Theorem 1. (Ryser [19]) The reconstruction problem with projections $H, V$ has a solution if and only if $H \leq_{d} V^{*}$, where $V^{*}=\left(v_{1}^{*}, v_{2}^{*}, \ldots, v_{n}^{*}\right)$ is defined as $v_{i}^{*}=\left|\left\{v_{j}: v_{j} \geq i\right\}\right|$. The problem admits a unique solution if and only if $H=V^{*}$.

In this section we formally define the reconstruction problem involving blocking components. For a simpler notation, we define a partition of the cells of a matrix $A$ in three different submatrices $C, X$ and $Y$ in order to be able to identify immediately the relative position of each cell with a blocking component. These three matrices are dependent from $A$, however we omit this dependency in the notation. The cells in $C$ are those who give contribution to both the horizontal and vertical projections, while those in $X$ and $Y$ are only counted in one of the two. We have:

$-C=\left(c_{i, j}\right)$ of size $\left(n-k^{v}\right) \times\left(m-k^{h}\right)$ with $c_{i, j}=a_{i, j}$ for $i \leq n-k^{v}$ and $j \leq m-k^{h}$;

$-X=\left(x_{i, j}\right)$ of size $\left(n-k^{v}\right) \times k^{h}$ with $x_{i, j}=a_{i, m-k^{h}+j}$ for $1 \leq j \leq k^{h}$;

- $Y=\left(y_{i, j}\right)$ of size $k^{v} \times\left(m-k^{h}\right)$ with $y_{i, j}=a_{n-k^{v}+i, j}$ for $1 \leq i \leq k^{v}$.

We define the operators $H(M)$ and $V(M)$ that extract the horizontal and vertical projections of a generic matrix $M$. We use the following operators to count the number of ones in each zone; we define $N^{C}(A)$ as $\sum c_{i, j}$, and similarly we refer to $N^{X}(A)$ and $N^{Y}(A)$; also $N(A)=\sum_{a_{i, j} \neq *} a_{i, j}$. When the argument of these operators is unambiguous, we omit the argument $(A)$ of the function, referring simply, for example, to $N^{C}$ or $N^{X}$. Finally, $N^{H}=\sum_{i} h_{i}$ and $N^{V}=$ $\sum_{j} v_{j}$

One of the additional difficulties of our problem is that the number of cells in $H$ may be different from the ones in $V$, since some cells contribute only one of the horizontal or vertical projections. In order to define a reconstruction algorithm, one of the first steps is to determine correct values for $N^{X}$ and $N^{Y}$. As some first trivial conditions, it must stand $N^{C}+N^{X}+N^{Y}=N$, and $N^{Y} \geq D$. The maximal number of cells $M^{X}$ (symmetrically, $M^{Y}$ ) in the group $X$ (resp. $Y$ ) for any solution of the problem is given by the following property.

Property 1. For any matrix $A$ having $V$ as vertical projections, we have $N^{Y} \leq$ $M^{Y}$, where $M^{Y}$ is defined by $M^{Y}=\sum_{j} \min \left(v_{j}, k^{v}\right)$.

Proof. To prove this simple property, it is sufficient to observe that $N^{Y}>M^{Y}$ would imply that on some column the number of cells equal to 1 would exceed the height $k^{v}$ of the matrix $Y$ or a vertical projection, bringing to a contradiction. 
Note that while the $N$ operators have as argument a matrix, the $M$ operators are in dependence from the input of the problem. From this property and the trivial condition $N^{Y}=N^{X}+D$ we obtain the following corollary.

Property 2. For any matrix $A$ solution of a reconstruction problem with blocking component, we have $N^{Y} \leq \min \left(M^{Y}, M^{X}+D\right)$.

\subsection{Switching Components and Unique Solutions}

We recall that the cells inside the blocking component of a matrix $A$, i.e. the elements $a_{i, j}$ such that $i>n-k^{v}$ and $j>m-k^{h}$, are represented with the special symbol $*$. We now define some switching operations that, starting from a solution of a problem, enable us to build other matrices satisfying the problem constraints. These operations are similar to the standard switches found in literature, but the presence of a blocking component leads to other types of switches involving the symbol $*$. The possible switches are shown in Figure 2 (left); it is easy to verify that all these operations do not alter the horizontal and vertical projections of a matrix.
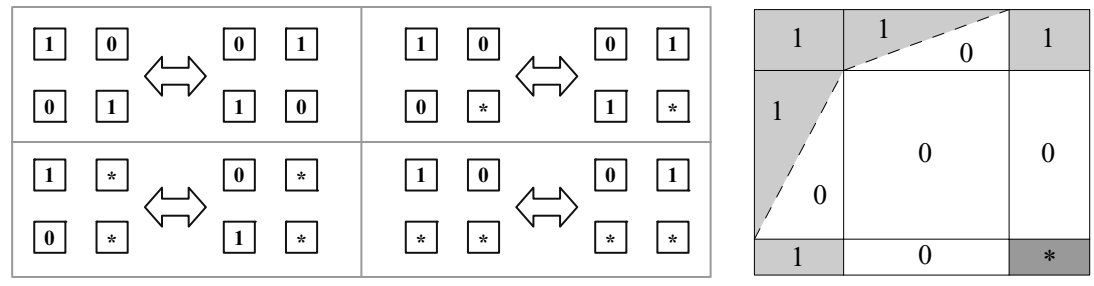

Fig. 2. (Left) Possible switches in our instances. The one in the upper left is the classical switching operation in the standard reconstruction problem, while the other three are introduced by the existence of a blocking component in the problem. (Right) The only possible structure of a matrix with no switching components, assuming rows and columns are ordered by projections.

Considering these four types of switch, we can state a uniqueness result. In that aim, we first show the structure of a matrix $A$ that does not contain any of the switching components. We recall that since w.l.g. $h_{1} \geq h_{2} \geq \ldots$ and $v_{1} \geq v_{2} \geq \ldots$, then we have:

Theorem 2. Let $A$ be a solution of a blocking component reconstruction problem. The matrix $A$ does not contain any switching components if and only if its partitions $C, X$ and $Y$ satisfy the conditions below, for the generic values $i \in[1, n]$ and $j, j^{\prime} \in[1, m]$.

1. $\forall i, j, j^{\prime}$ then $x_{i, j}=x_{i, j^{\prime}}$;

2. $\forall i, i^{\prime}, j$ then $y_{i, j}=y_{i^{\prime}, j}$; 
3. $\forall x_{i, j}=y_{i^{\prime}, j^{\prime}}$ then $c_{i, j^{\prime}}=x_{i, j}=y_{i^{\prime}, j^{\prime}}$;

4. $H(C)=V^{*}(C)$.

Proof. Observe that to avoid switching components involving two equal symbols * every row of $X$ and every column of $Y$ must contain only one of the values (points 1 and 2 ). To prohibit switches with one ${ }^{*}$, we impose Condition 3 , while rule 4 must be adopted in order to prevent the standard switching operation.

In Figure 2 (right) we depicted a graphical representation of a matrix $A$ without any switching components. An example of a unique solution on a specific problem instance can be seen in Figure 3 .

In the standard reconstruction problem, the absence of switching components is sufficient to prove that a matrix is the unique solution. In this problem, however, we may speculate about this fact, as two different solutions may exist, but that there is no series of switching operations that transform one of them into the other. The following theorem is necessary to show how this conjecture turns out to be false, and furthermore its proof exhibits a procedure allowing the determination of the unique solution if it exists.

Theorem 3. If a matrix with no switching components exists for a blocking component reconstruction problem, then the given matrix is the only solution of the problem.

Proof. Consider as input of the problem the dimension of the blocking component described by $k^{v}$ and $k^{h}$ and the vectors of projections $H=\left(h_{1}, \ldots, h_{n-k^{v}}\right)$, $V=\left(v_{1}, \ldots, v_{m-k^{h}}\right)$. We shall determine, if possible, a value $s \in\left[0, v_{1}-k^{v}\right]$ such that for the $k^{v}$ elements in $V^{*}$ from $v_{s+1}^{*}$ to $v_{s+k^{v}}^{*}$, we have $v_{s+1}^{*}=\ldots=v_{s+k^{v}}^{*}=$ $r$ for some value $r$, and such that $H=\left(v_{1}^{*}+k^{h}, \ldots, v_{s}^{*}+k^{h}, v_{s+k^{v}+1}^{*}, \ldots, v_{v_{1}}^{*}\right)$. The solution for the blocking component reconstruction problem is given by a matrix $A$ whose submatrices are defined uniquely by the following:

- $H(C)=V^{*}(C)=\left(v_{1}^{*}, \ldots, v_{s}^{*}, v_{s+k^{v}+1}^{*}, \ldots, v_{v_{1}}^{*}\right)$;

- $H(X)=\left(x_{1}, \ldots, x_{s}\right)$ where $x_{i}=k^{h}, 1 \leq i \leq s$

- $V(Y)=\left(y_{1}, \ldots, y_{r}\right)$ where $y_{j}=k^{v}, 1 \leq j \leq r$.

Clearly, $H(C)+H(X)=H$ and $V(C)+V(Y)=V$; note that knowing $H(X)$ and $V(Y)$ the matrices $X$ and $Y$ are trivially determined. By Theorem 2 each solution with the previous structure does not admit any switching components. Supposing that such solution exists, then we show that it is unique by proving that there is only one possible value for $s \in\left[0, v_{1}-k^{v}\right]$. Let us compute the number of ones in the matrices $C$ and $X$ :

$$
\sum_{i=1}^{v_{1}} v_{i}^{*}-\sum_{i=s}^{s+k^{v}} v_{i}^{*}+s k^{h}=\sum_{i=1}^{n-k^{v}} h_{i} .
$$

Since $V^{*}$ is decreasing, then $\sum_{i=s}^{s+k^{v}} v_{i}^{*}$ is decreasing in $s$, and further $s k^{h}$ grows with $s$. From these properties, the left side of the equation is strictly increasing with $s$, so this variable can have only one value to satisfy the equation. 
In Figure 3 we depicted a graphical representation of the unique solution for the blocking component reconstruction problem having as input $k^{v}=3, k^{h}=2$, $H=(10,8,7,7,6,6,5,2,2,1,1)$ and $V=(14,12,10,6,4,2,1,1)$.

\section{The Reconstruction Algorithm}

In this section we describe the reconstruction algorithm for a problem with a blocking component. The core of the procedure is defined by Algorithm 1 that determines the vector $V(Y)$ given a fixed number $N^{Y}=\sum_{i} v_{i}(Y)$. We remark that the array $V(Y)$ contains the vertical projections of $Y$. Our goal is to define $V(Y)$ such that the vector $V-V(Y)$ is the minimum that we can obtain with respect to the dominance ordering; this property guarantees that if $V-V(Y)$ and $H-H(X)$ are not consistent with a solution for the standard reconstruction problem for matrix $C$, then any other configuration of $X$ and $Y$ cannot yield a solution. Without loss of generality, we impose that the elements of both $V$ and $V-V(Y)$ are ordered. The idea of the procedure consists in determining a value $p$ such that for every element $v_{i} \geq p$ we may set $v_{i}(Y) \in\left\{k^{v}, v_{i}-p, v_{i}-p+1\right\}$, and for every element $v_{i}<p$ then $v_{i}(Y)=0$. To compute $p$, the procedure uses a vector $T$, containing the maximum number of cells that could be contained in $V(Y)$ for every possible choice of $p$. Doing so, placing $k^{v}$ elements in the first columns of $V(Y)$ and 0 in the last ones, we maximize the vector $V-V(Y)$ in the dominance ordering. In Figure 4 is shown an example of the execution of Algorithm 1, where $k^{v}=3$ and $N^{Y}=23$.

It may be possible to compute the vectors $T$ and $Z$ with a closed formula, but this algorithmic formulation simplifies the following proof.

Theorem 4. Consider a vector $V$ and two fixed integers $N^{Y}$ and $k^{v}$, and call $V-V(Y)$ the output of Algorithm 1 with input $V, N^{Y}, k^{v}$. For any ordered
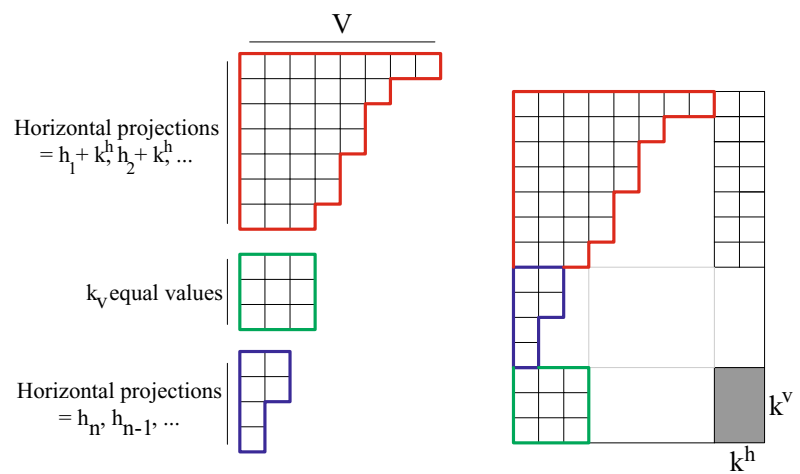

Fig. 3. Computing the only solution without switching components. To the left, we have the graphical representation of $V$, while to the right we can see the unique solution of the problem. 


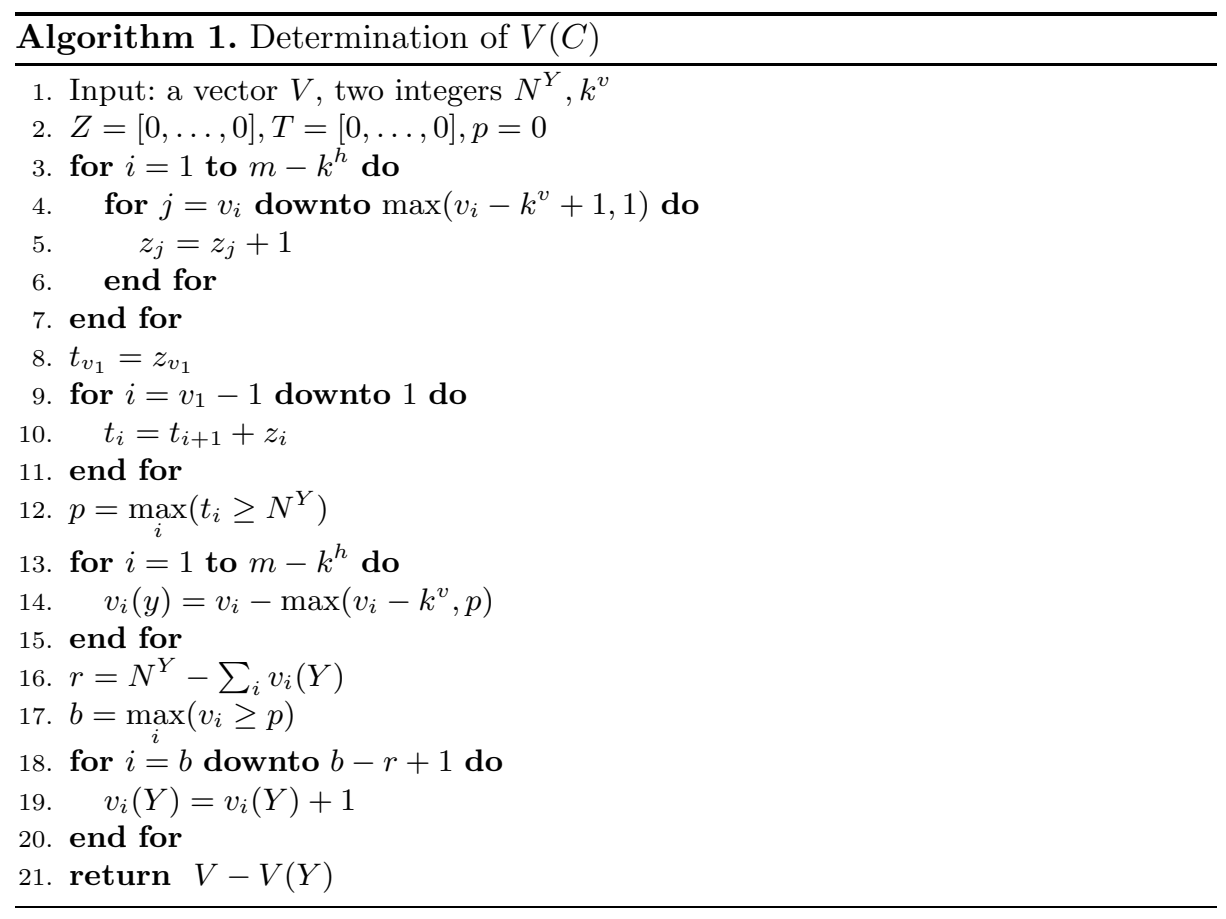

vector $V^{\prime}(Y)$ such that $\sum_{i} v_{i}^{\prime}(Y)=\sum_{i} v_{i}(Y)$ and $\forall i, v_{i}^{\prime}(Y) \leq \min \left(k^{v}, v_{i}\right)$, we have $V-V(Y) \leq_{d} V-V^{\prime}(Y)$.

Proof. (Sketch) Call $K=V-V(Y)$ and $L=V-V^{\prime}(Y)$, and suppose by contradiction that it does not stand that $K \leq_{d} L$, hence that for some index $i, \sum_{w=1}^{i} k_{w}>\sum_{w=1}^{i} l_{w}$. Since $\sum_{w} k_{w}=\sum_{w} l_{w}$ this implies that for some $j>$ $i, k_{j}<l_{j}$. Consider the value $p$ computed in the procedure, and name $a=$ $\max _{w}\left(v_{w}>p+k_{w}\right)$ and $b=\min _{w}\left(v_{w}<p\right)$ (b is the same value as the one computed in Algorithm 1). For $w \leq a$ then $V(Y)=k^{v}$, hence $i>a$. For $w \geq b$, then $V(Y)=0$, and $i<j<b$. By construction of the algorithm, it follows that $\left[k_{a+1}, \ldots, k_{b-1}\right]=[p, \ldots, p, p-1, \ldots, p-1]$, hence no indexes $i$ and $j$ can be found in this interval that satisfy the conditions $\sum_{w=1}^{i} k_{w}>\sum_{w=1}^{i} l_{w}$ and $k_{j}<l_{j}$, and also maintain the vector $K$ ordered, hence this brings us to our contradiction.

At this point we are ready to define formally our reconstruction procedure; since we have proved that given a fixed $N^{Y}$, Algorithm 1 executes an optimal choice for the cells in $V(Y)$ (resp. $H(X)$ ). Thanks to this property, to solve the problem it would suffice to find an appropriate value for $N^{Y}\left(\operatorname{resp} . N^{Y}-D\right)$. Unfortunately, we have not found yet a compact formula to determine this value. For this reason, the following procedure, described by Algorithm 2, iterates on all possible values until one yielding a solution is found. 


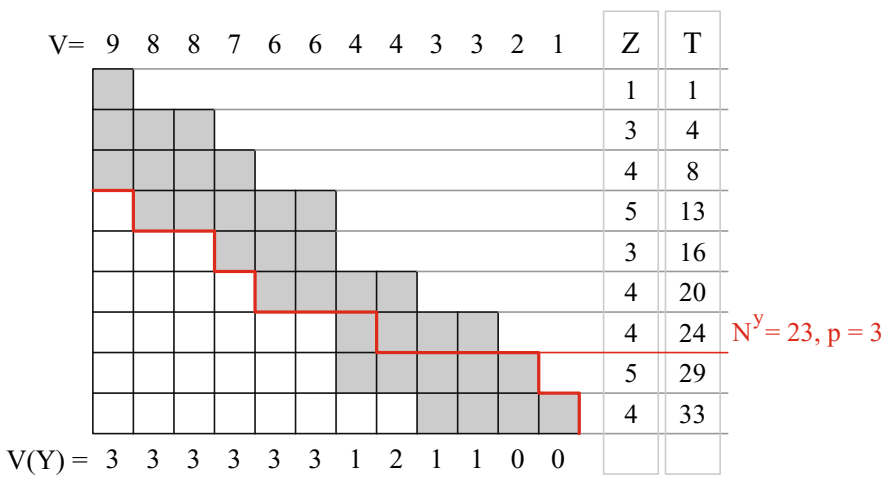

Fig. 4. The optimal choice for vector $V(Y)$; in grey, the maximum number of cells that could be placed in each column given $k^{v}=3$. In this example, $p=3$, hence every grey cell at height $>3$ contributes to $V(Y)$, while every cell of height $<3$ doesn't.

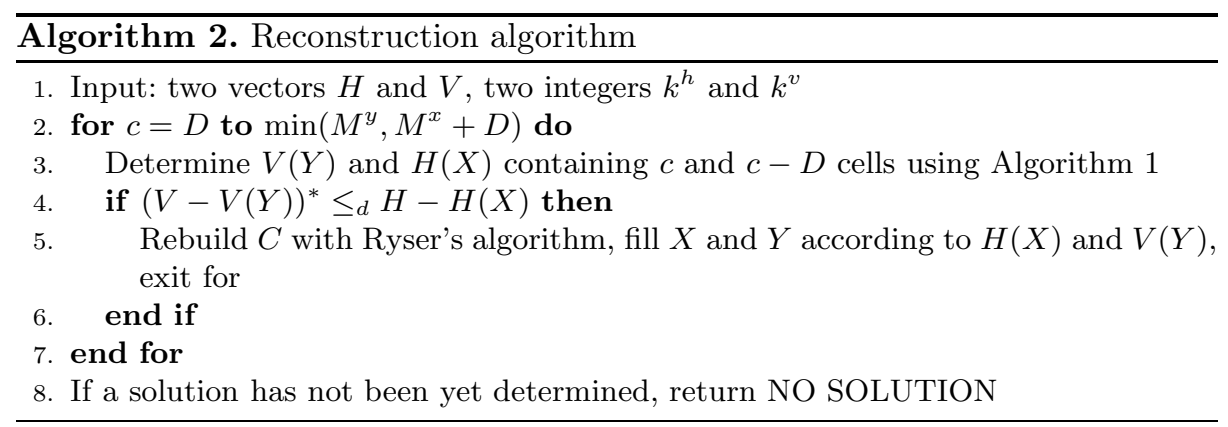

\section{A Network Flow Approach to Reconstruction}

In this section, we show how the blocking component reconstruction problem may be solved by means of a reduction to a network flow problem. It is well known that the standard reconstruction problem can be solved by a network flow approach; the procedure consists in considering a source for every $h_{i}$ with capacity equal to the projection, a sink for every $v_{j}$ again with the appropriate capacity, and a node for every cell $(i, j)$ connected to the related source and sink with arcs of capacity 1.

Beyond showing an interesting connection with an important research field, this reduction also enables us to define a simple algorithm to solve the problem in the case where we have some forbidden positions, i.e. cells that can not have a value of 1 . This can be useful in a variety of cases in applications, for example if we already know the configuration of some areas of the image and we want to include this information in the solution; note that positions where a cell must have value 1 can be included in the formulation of the problem with forbidden 
positions, by simply subtracting 1 from the related projections and setting the cell to a forbidden position. Using the network flow equivalence, we can solve the problem by simply removing the nodes deriving from forbidden positions. Hence by giving a similar reduction in our problem, we also trivially solve the blocking component reconstruction problem with forbidden positions.

The idea of the construction is to compensate the difference in the projections $D$ with an artificial source. Since at least $D$ cells of $Y$ must be equal to 1 in order to obtain a solution, we connect the source with capacity $D$ to all of the cells in $Y$; further, since for every cell $x_{i, j}=1$, there must be an additional cell in $Y$, we also connect the cells in $X$ to every cell in $Y$.

Formally, the flow problem is composed by the following elements. To simplify the notation, we refer to a node with the name of the related cell; every pair of connected nodes has an edge of capacity 1 linking them. We call the following problem the associate flow problem of the original reconstruction one:

- $|H|$ sources of capacity $h_{1}, \ldots, h_{n-k^{v}}$;

- 1 source of capacity $D$;

- $|V|$ sinks of capacity $v_{1}, \ldots, v_{m-k^{h}}$;

- $|C|$ nodes, where $c_{i, j}$ is connected with source $h_{i}$ and sink $v_{j}$;

- $|Y|$ nodes, where $y_{i, j}$ is connected to the source $D$, to the sink $v_{j}$ and with every node in $X$;

- $|X|$ nodes, where $x_{i, j}$ is connected to each source $h_{i}$ and with every node in $Y$.

The problem configuration is depicted in Figure 5, where the cells are represented by groups, and an edge labelled $r$ represents a series of edges connecting all nodes related by a column or row (as $c_{i, j}$ with $h_{i}$ ) while unlabelled edges represent connections between all possible pairs of the two groups.

Theorem 5. A reconstruction problem $P$ admits a solution if and only if the associate flow problem $F$ has a solution.

Proof. (Sketch) From a solution of the flow problem, we can build a matrix that is solution of $P$ by simply setting to 1 every cell in $F$ where the flow enters and

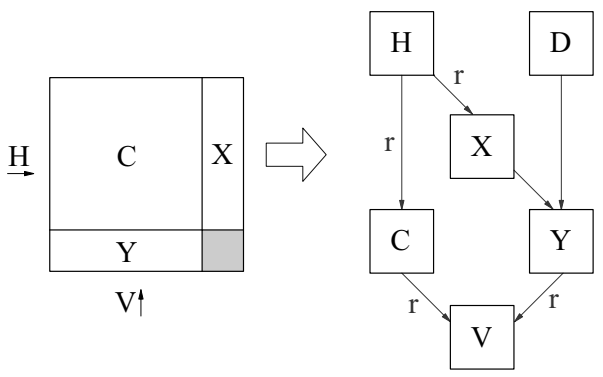

Fig. 5. Reducing an instance of the reconstruction problem to a flow problem 
exits. It is easy to verify that the projections match the input vectors $H$ and $V$, as in $F$ every sink and source receives (emits) a quantity of flow equivalent to the contribution of the cell to the related projection.

Conversely, starting from a solution of $P$, we can build a solution of $F$ in a similar fashion, but while the edges connecting the groups $H, C$ or $C, V$ or $H, X$ are uniquely determined, we may have many ways to connect the cells in $X, Y$ and $D, Y$. Any choice yields a correct solution, as long as the number of cells to select in the $Y$ group is equal to $D$ plus the number of nodes to select in the $X$ group, and this property follows immediately from the definition of $D$.

\section{Conclusions}

We have studied how the standard reconstruction problem in discrete tomography can be extended to the case where some vertical and horizontal projections are unavailable, as if a component of the scanned object blocked the x-rays used to study the material. We defined a criteria to determine when a problem has a unique solution, and we furnished two polynomial reconstruction algorithms. One allows us to reduce the problem to the standard reconstruction, allowing the usage of the efficient algorithms known in literature; the other transforms the problem in a flow problem, allowing us to solve it even when we want some prescribed values in the output image.

Since the existence of a blocking component is a recurring problem in some applications, in future works it will be interesting to consider also other assumptions on the image that has to be rebuilt, describing realistic environments. For example, it would be interesting to study how a reconstruction algorithm could work if the image must represent a convex polyomino as in 4, or more generally if we have of some skeletal information as in [15]. Introducing the right assumptions, this line of research could indeed obtain results that could be applied in tomographic applications dealing with reconstruction artifacts caused by blocking components.

\section{References}

1. Barcucci, E., Brlek, S., Brocchi, S.: PCIF: an algorithm for lossless true color image compression. In: Wiederhold, P., Barneva, R.P. (eds.) IWCIA 2009. LNCS, vol. 5852, pp. 224-237. Springer, Heidelberg (2009)

2. Barcucci, E., Brocchi, S.: Solving multicolor discrete tomography problems by using prior knowledge. Fundamenta Informaticae 125, 313-328 (2013)

3. Barcucci, E., Brocchi, S., Frosini, A.: Solving the two color problem - An heuristic algorithm. In: Aggarwal, J.K., Barneva, R.P., Brimkov, V.E., Koroutchev, K.N., Korutcheva, E.R. (eds.) IWCIA 2011. LNCS, vol. 6636, pp. 298-310. Springer, Heidelberg (2011)

4. Barcucci, E., Del Lungo, A., Nivat, M., Pinzani, R.: Reconstructing convex polyominoes from horizontal and vertical projections. Theoretical Computer Science 155, 321-347 (1996) 
5. Batenburg, K.J.: A network flow algorithm for reconstructing binary images from discrete X-rays. Journal of Mathematical Imaging and Vision 27(2), 175-191 (2013)

6. Batenburg, K.J., Sijbers, J.: DART: a practical reconstruction algorithm for discrete tomography. IEEE Transactions on Image Processing 20(9), 2542-2553 (2011)

7. Costa, M.-C., de Werra, D., Picouleau, C., Schindl, D.: A solvable case of image reconstruction in discrete tomography. Discrete Applied Mathematics 148(3), 240$245(2005)$

8. De Man, B., Nuyts, J., Dupont, P., Marchal, G., Suetens, P.: Reduction of metal streak artifacts in x-ray computed tomography using a transmission maximum a posteriori algorithm. In: Nuclear Science Symposium, pp. 850-854 (1999)

9. Duan, X., Zhang, L., Xiao, Y., Cheng, J., Chen, Z., Xing, Y.: Metal artifact reduction in CT images by sinogram TV inpainting. In: Nuclear Science Symposium Conference, pp. 4175-4177 (2008)

10. Durr, C., Guinez, F., Matamala, M.: Reconstructing 3-Colored Grids from Horizontal and Vertical Projections is NP-Hard, A Solution to the 2-Atom Problem in Discrete Tomography. SIAM J. Discrete Math. 26(1), 330-352 (2012)

11. Faggiano, E., Lorenzi, T., Quarteroni, A.: Metal Artifact Reduction in Computed Tomography Images by Variational Inpainting Methods, MOX-report No. 16/2013 (2013)

12. Gardner, R.J., Gritzmann, P., Pranenberg, D.: On the computational complexity of reconstructing lattice sets from their X-rays. Discrete Mathematics 202(1-3), 45-71 (1999)

13. Guinez, F.: A formulation of the wide partition conjecture using the atom problem in discrete tomography. Discrete Applied Mathematics (2013) (in press)

14. Hantos, N., Balazs, P.: A uniqueness result for reconstructing hv-convex polyominoes from horizontal and vertical projections and morphological skeleton. In: 8th International Symposium on Image and Signal Processing and Analysis (ISPA). IEEE (2013)

15. Hantos, N., Balazs, P.: The Reconstruction of Polyominoes from Horizontal and Vertical Projections and Morphological Skeleton is NP-complete. Fundamenta Informaticae 125(3), 343-359 (2013)

16. Herman, G., Kuba, A.: Advances in discrete tomography and its applications. Birkhauser, Boston (2007)

17. Kalender, W.A., Hebel, R., Ebersberger, J.: Reduction of CT artifacts caused by metallic implants. Radiology 164(2), 576-577 (1987)

18. Picouleau, C., Brunetti, S., Frosini, A.: Reconstructing a binary matrix under timetabling constraints. Electronic Notes in Discrete Mathematics 20, 99-112 (2005)

19. Ryser, H.J.: Combinatorial properties of matrices of zeros and ones. Canadian Journal of Mathematics 9, 371-377 (1957)

20. Wang, G., Snyder, D.L., O'Sullivan, J., Vannier, M.: Iterative deblurring for ct metal artifact reduction. IEEE Transactions on Medical Imaging 15(5), 657-664 (1996) 